\title{
Inflammation, Amyloid, and Atrophy in The Aging Brain: Relationships with Longitudinal Changes in Cognition
}

\author{
Roser Sala-Llonch $^{\mathrm{a}, \mathrm{b}, *}$, Ane-Victoria Idland ${ }^{\mathrm{a}, \mathrm{c}}$, Tom Borza ${ }^{\mathrm{c}, \mathrm{d}}$, Leiv Otto Watne ${ }^{\mathrm{c}, \mathrm{e}}$, \\ Torgeir Bruun Wyller ${ }^{\mathrm{c}, \mathrm{f}}$, Anne Brækhus ${ }^{\mathrm{f}, \mathrm{g}, \mathrm{h}}$, Henrik Zetterberg ${ }^{\mathrm{i}, \mathrm{j}, \mathrm{k}}$, Kaj Blennow ${ }^{\mathrm{i}, \mathrm{j}}$, \\ Kristine Beate Walhovd ${ }^{\mathrm{a}}$ and Anders Martin Fjell ${ }^{\mathrm{a}}$ \\ ${ }^{a}$ Department of Psychology, Center for Lifespan Changes in Brain and Cognition, University of Oslo, \\ Oslo, Norway \\ ${ }^{\mathrm{b}}$ Department of Biomedicine, Faculty of Medicine, University of Barcelona, Barcelona, Spain \\ ${ }^{\mathrm{c}}$ Institute of Clinical Medicine, Faculty of Medicine, University of Oslo, Oslo, Norway \\ ${ }^{\mathrm{d}}$ Centre for Old Age Psychiatric Research, Innlandet Hospital Trust, Ottestad, Norway \\ ${ }^{\mathrm{e}}$ Institute of Basic Medical Sciences, University of Oslo, Oslo, Norway \\ ${ }^{\mathrm{f}}$ Department of Geriatric Medicine, Oslo University Hospital, Oslo, Norway

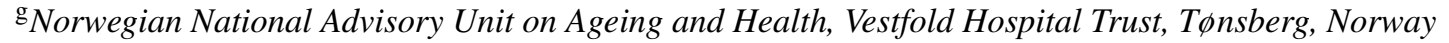 \\ ${ }^{\mathrm{h}}$ Department of Neurology, Oslo University Hospital, Oslo, Norway \\ i Department of Psychiatry and Neurochemistry, Institute of Neuroscience and Physiology, \\ The Sahlgrenska Academy at University of Gothenburg, Gothenburg, Sweden \\ ${ }^{\mathrm{j}}$ Clinical Neurochemistry Laboratory, Sahlgrenska University Hospital, Mölndal, Sweden \\ ${ }^{\mathrm{k}}$ Department of Molecular Neuroscience, UCL Institute of Neurology, Queen Square, London, UK
}

\begin{abstract}
Amyloid deposition occurs in aging, even in individuals free from cognitive symptoms, and is often interpreted as preclinical Alzheimer's disease (AD) pathophysiology. YKL-40 is a marker of neuroinflammation, being increased in AD, and hypothesized to interact with amyloid- $\beta(A \beta)$ in causing cognitive decline early in the cascade of AD pathophysiology. Whether and how $\mathrm{A} \beta$ and YKL-40 affect brain and cognitive changes in cognitively healthy older adults is still unknown. We studied 89 participants (mean age: 73.1 years) with cerebrospinal fluid samples at baseline, and both MRI and cognitive assessments from two time-points separated by two years. We tested how baseline levels of $A \beta_{42}$ and YKL-40 correlated with changes in cortical thickness and cognition. Thickness change correlated with $A \beta_{42}$ only in $A \beta_{42}+$ participants $(<600$ $\mathrm{pg} / \mathrm{mL}, n=27)$ in the left motor and premotor cortices. $\mathrm{A} \beta_{42}$ was unrelated to cognitive change. Increased YKL-40 was associated with less preservation of scores on the animal naming test in the total sample $(r=-0.28, p=0.012)$ and less preservation of a score reflecting global cognitive function for $\mathrm{A} \beta_{42}+$ participants $(r=-0.58, p=0.004)$. Our results suggest a role for inflammation in brain atrophy and cognitive changes in cognitively normal older adults, which partly depended on $\mathrm{A} \beta$ accumulation.
\end{abstract}

Keywords: Aging, biomarkers, cerebrospinal fluid, cognition, cortical thickness, inflammation, memory, MRI, YKL-40

${ }^{*}$ Correspondence to: Roser Sala-Llonch, PhD, Department of Psychology, Center for Lifespan Changes in Brain and Cognition, University of Oslo, Oslo, Norway. Harald Schjelderups Hus, Forskningsveien 3A, 0373 Oslo, Norway. Tel.: +47 22845129; E-mail: r.s.llonch@psykologi.uio.no.

\section{INTRODUCTION}

Low level of cerebrospinal fluid (CSF) amyloid$\beta 42\left(\mathrm{~A} \beta_{42}\right)$ represents a major risk factor for Alzheimer's disease (AD) [1] but it is also a commonly described feature within clinically normal 
aging populations [2]. Numerous studies show high concordance between low CSF $A \beta_{42}$ and high ligand retention on amyloid positron emission tomography (PET), irrespective of clinical status [3] indicating that low CSF $A \beta_{42}$ reflects amyloid deposition. However, we still do not understand why $A \beta_{42}$ is related to the development of disease in some persons, while others seem able to cope with substantial amyloid burden. Therefore, many studies point to an inconsistent relationship between $A \beta_{42}$, brain atrophy as measured with magnetic resonance imaging (MRI), and cognition in aging, which can be caused by other factors that interact with amyloid [4].

In the sense of these interactions with amyloid, neuroinflammation has been proposed as one critical factor. Similar to amyloid deposition, neuroinflammation occurs in both $\mathrm{AD}$ and normal aging $[5,6]$. Although neuroinflammation is observed in pathologically vulnerable brain regions in $A D$, it remains unclear whether insoluble $A \beta$ deposits or neurofibrillary tangles are causing inflammation [7] or whether inflammation may actually promote or accelerate deposition of $A \beta$ [8]. In addition, by means of neuropathological studies, we know that increasing age is associated with increased microglial activation accompanied by production of inflammatory cytokines and compromised neuronal function, including synaptic dysfunction [9]. The chitinase-3like protein 1 (YKL-40) is a CSF biomarker reflecting neuroinflammation [10]. Some studies have reported higher YKL-40 levels in AD [11], and these increases have also been associated with conversion to dementia $[10,12,13]$. YKL-40 has also been related to brain atrophy in $\mathrm{AD}[14,15]$. However, the capabilities of YKL-40 to distinguish AD from controls have so far been found to be moderate in comparison with other CSF-markers such as total tau (T-tau), phosphorylated tau (P-tau), and $\mathrm{A} \beta_{42}$ [16], and some report no relationship with $\mathrm{AD}[17,18]$. This may suggest that YKL-40 levels correlate with brain and cognitive processes just as much in normal aging as in AD.

In the present study, we tested the role of neuroinflammation in cortical atrophy and cognitive change in older adults without cognitive symptoms with either abnormal or normal amyloid status. For this purpose, we used baseline measures of $A \beta_{42}$ and YKL-40, a measure of the brain cortical thickness and the scores of cognitive tests. Although there are reasons to expect a relationship between amyloid levels and neuroinflammation, no previous study has directly tested how these biomarkers interact in contributing to brain and cognitive decline in aging. While the role of YKL-40 in normal aging is almost unexplored, previous research on amyloidrelated changes in the aging brain are conflicting, with some reporting more atrophy [19-21] in subjects with lower CSF $A \beta_{42}$ levels and others larger volumes [22], or non-linear relationships [23]. It has also been suggested that the correlations between $A \beta_{42}$ and atrophy can be different for $A \beta_{42}$ positive versus negative individuals. This has been described in the Alzheimer's Disease Neuroimaging Initiative (ADNI) database [24, 25], but has to our knowledge not been tested in other samples. With regard to cognitive changes, studies indicate that amyloid levels correlate, albeit not strongly, with cognitive function in normal populations [26]. Because of these controversies, it is necessary to study other factors that may show either amyloid-independent or amyloid-dependent relationships to atrophy and cognitive changes.

\section{MATERIALS AND METHODS}

\section{Participants}

The sample consisted of cognitively healthy older adults undergoing elective spinal surgery under spinal anesthesia and turning 65 years or older the year of inclusion. Exclusion criteria were dementia, previous stroke with sequelae, Parkinson's disease, or other neurological condition likely to affect cognition. From the full sample recruited $(n=172)$, we selected those having available baseline CSF measures and both MRI and cognitive evaluation at the two time points (mean time between MRI scans: 2.18 years). None developed mild cognitive impairment at follow up. A total of 89 participants were included in the current analyses. Demographics, including years of education, are summarized in Table 1 . The cognitive evaluation was identical at the two time points and included the Word List Memory Task from the Consortium to Establish a Registry for Alzheimer's Disease battery (CERAD) [27], which was based on recall, the Animal Naming Test (ANT) [28] and the Trail Making Test B (TMTB) [29]. These tests represent verbal memory, verbal fluency, and executive function. We also report here the results of the Mini-Mental Status Examination (MMSE) [30]. In addition, the Clock Drawing Test [31] and Kendrick Object Learning Test [32] were 
Table 1

Demographics, cognitive, and CSF data of the participants included in the analyses

\begin{tabular}{|c|c|c|c|}
\hline & $\begin{array}{c}\text { Full sample } \\
\mathrm{N}=89 \\
\text { Mean (SD) } \\
\text { Range }\end{array}$ & $\begin{array}{c}\mathrm{A} \beta-\text { group } \\
\mathrm{N}=62 \\
\text { Mean }(\mathrm{SD}) \\
\text { Range }\end{array}$ & $\begin{array}{c}\mathrm{A} \beta+\text { group } \\
\mathrm{N}=27 \\
\text { Mean }(\mathrm{SD}) \\
\text { Range }\end{array}$ \\
\hline \multicolumn{4}{|l|}{ Sample \& Demographics } \\
\hline \multirow[t]{2}{*}{ Age (years) } & $73.1(6.01)$ & $73.2(6.3)$ & $72.91(5.38)$ \\
\hline & $64.74-89.79$ & $64.74-89.79$ & $64.95-82.52$ \\
\hline Sex (men/women) & $46 / 43$ & $29 / 33$ & $17 / 10$ \\
\hline \multirow[t]{2}{*}{ Time between (years) } & $2.17(0.28)$ & $2.18(0.26)$ & $2.17(0.33)$ \\
\hline & $1.55-2.88$ & $1.55-2.87$ & $1.71-2.88$ \\
\hline \multirow[t]{2}{*}{ MMSE baseline } & $29.13(1.27)$ & $29.09(1.40)$ & $29.22(0.93)$ \\
\hline & $25-30$ & $25-30$ & $27-30$ \\
\hline \multirow[t]{2}{*}{ MMSE 2 years } & $29.05(1.21)$ & $29.14(1.19)$ & $28.85(1.23)$ \\
\hline & $24-30$ & $24-30$ & $26-30$ \\
\hline \multirow[t]{2}{*}{ Education (years) } & $14.49(3.34)$ & $14.82(3.31)$ & $13.72(3.35)$ \\
\hline & $8-23$ & $8-23$ & $8-19$ \\
\hline \multirow[t]{2}{*}{ WMH baseline $\left(\mathrm{mm}^{3}\right)$} & $6224.45(7161.05)$ & $6553.5(7881)$ & $4946.4(4486.1)$ \\
\hline & $1143.4-36056.6$ & $1143.40-36056.6$ & $1338.2-22018.5$ \\
\hline \multirow[t]{2}{*}{ WMH 2 years $\left(\mathrm{mm}^{3}\right)$} & $7091.84(8343.7)$ & $7670.9(9365.2)$ & $5777.7(5277.05)$ \\
\hline & $1098.9-40698.6$ & $1098.9-40698.6$ & $1611.7-24642.6$ \\
\hline \multirow{2}{*}{ WMH change $\left(\mathrm{mm}^{3}\right)$} & $0.105(0.098)$ & $0.103(0.100)$ & $0.108(0.093)$ \\
\hline & $-0.28-0.45$ & $-0.28-0.45$ & $-0.09-0.26$ \\
\hline \multicolumn{4}{|l|}{ Cognition } \\
\hline \multirow[t]{2}{*}{ TMTB - baseline ${ }^{+}$} & $118.55(60.28)$ & $112.61(44.33)$ & $132.69(64.13)$ \\
\hline & $34-466$ & $34-246$ & 64-466 \\
\hline \multirow[t]{2}{*}{ TMTB 2 years $^{+}$} & $116.23(56.57)$ & $106.64(50.53)$ & $137.89(64.13)$ \\
\hline & $31-306$ & $31-267$ & $72-306$ \\
\hline \multirow[t]{2}{*}{ CERAD baseline } & $6.78(1.76)$ & $6.73(1.67)$ & $6.89(2)$ \\
\hline & $3-10$ & $4-10$ & $3-10$ \\
\hline \multirow[t]{2}{*}{ CERAD 2 years } & $7.69(1.72)$ & $7.61(1.55)$ & $7.85(2.1)$ \\
\hline & $3-10$ & $5-10$ & $3-10$ \\
\hline \multirow[t]{2}{*}{ ANT baseline } & $20.67(5.45)$ & $20.69(5.22)$ & $20.63(6.04)$ \\
\hline & $5-32$ & $10-32$ & $5-30$ \\
\hline \multirow[t]{2}{*}{ ANT 2 years } & 23.27 (6.09) & $23.85(5.9)$ & $21.93(6.4)$ \\
\hline & $7-38$ & $7-38$ & $10-38$ \\
\hline \multicolumn{4}{|l|}{ CSF biomarkers } \\
\hline \multirow[t]{2}{*}{$\mathrm{CSF}-\mathrm{A} \beta_{42}(\mathrm{pg} / \mathrm{ml})$} & $721.22(204.65)$ & 831.25 (131.07) & $467.19(72.45)$ \\
\hline & $275-1175$ & $605-1175$ & $275-587$ \\
\hline \multirow[t]{2}{*}{ CSF - T-Tau (pg/ml) } & $373.4(142.05)$ & $361.28(123.27)$ & $401.23(177.45)$ \\
\hline & $114.7-785.9$ & $181.8-767.9$ & $114.7-785.95$ \\
\hline \multirow[t]{2}{*}{ CSF - P-tau (pg/ml) } & $60.42(18.37)$ & $59.76(17.1)$ & $61.93(21.34)$ \\
\hline & $26-110$ & $32-110$ & $26-103$ \\
\hline \multirow{2}{*}{ YKL - $40(\mathrm{ng} / \mathrm{ml})^{*}$} & $220.02(73.9)^{*} 10^{3}$ & $219.80(73.39)^{*} 10^{3}$ & $220.521(76.47)^{*} 10^{3}$ \\
\hline & $92.11-441.5^{*} 10^{3}$ & $97.19-441.5^{*} 10^{3}$ & $92.11-348.3^{*} 10^{3}$ \\
\hline
\end{tabular}

MMSE, Mini-Mental State Examination; WMH, white matter hypointensities; TMTB, Trail Making Test - B; ANT, Animal Naming test; CERAD, Word list memory task from the consortium to establish a registry for Alzheimer's disease battery. ${ }^{+}$Missing data of $n=1$ subject; *excluded 1 subject below detection limit.

administered, but were not included here because they tend to be less sensitive to variation in cognitively healthy samples.

\section{Ethical considerations}

The study was conducted in accordance with the Declaration of Helsinki and approved by the Regional Committee for Ethics in Medical Research in Norway (REK 2011/2052). All participants provided written informed consent.

\section{Rates of change and cognition factor}

We computed the annual rate of change in the scores obtained from the cognitive tests using the symmetrized annual percent change. These values were obtained as the difference in scores between time points divided by its mean and by the time between them. The obtained scores are referred to as: CERAD-change, ANT-change, and TMTB-change.

We also calculated a global cognition change factor using principal component analysis on the rates 
of change obtained from the three tests. We used the first component, explaining the largest amount of variance. We refer to this score as COGfac-change.

\section{CSF measures}

CSF samples were taken at the time of the induction of spinal anesthesia. CSF was collected in polypropylene tubes, centrifuged, the supernatant aliquoted in polypropylene vials, frozen as soon as possible, and stored at $-80^{\circ} \mathrm{C}$ pending analyses. CSF levels of $\mathrm{A} \beta_{42}$, T-tau, and P-tau were determined using INNOTEST enzyme-linked immunosorbent assays (Fujirebio, Ghent, Belgium). YKL-40 concentrations were measured using a commercially available ELISA (R\&D systems, Minneapolis, MN). All analyses were performed by board-certified laboratory technicians, who were masked to clinical data, using one batch of reagents with intra-assay coefficients of variation below $10 \%$.

\section{Magnetic resonance imaging}

In each scanning session, a T1-weighted MPRAGE $3 \mathrm{D}$ image was acquired in a $1.5 \mathrm{~T}$ Siemens Avanto scanner using a 12-channel head coil $(T R=2400 \mathrm{~ms}$, $\mathrm{TE}=3.79 \mathrm{~ms}$, slice thickness $=1.20 \mathrm{~mm}$, pixel spacing $=1.25 \times 1.25 \mathrm{~mm}$ ).

\section{MRI processing}

Structural MPRAGE scans were processed with FreeSurfer (version 5.3) and its longitudinal stream (https://surfer.nmr.mgh.harvard.edu). The standard FreeSurfer pipeline performs a set of automated procedures for the cortical reconstruction and volumetric segmentation of the individual scans [33, 34]. In addition, the FreeSurfer longitudinal stream includes a set of methods designed to minimize the bias to any time point and which has been shown to lead to increased statistical power, better separation of groups based on atrophy and higher reproducibility [35-37]. Using these tools, we obtained vertex-wise maps of symmetrized atrophy rate, defined as the normalized difference in thickness at each vertex per year. FreeSurfer reconstructed brain surfaces were visually inspected and manually corrected when necessary. Manual interventions included the removal of nonbrain areas and the use of control points to guide the automated reconstruction of white and pial surfaces. We obtained the volume of WM hypointensities (WMH) from the automated freesurfer subcortical segmentation [34]. WMH appear as dark WM on the T1-weighted image and the overall WMH volume is obtained from the sum of regions within the WM with T1-intensity values within a certain range defined from the probabilistic atlas. This measure is related to WM lesions and vascular disease and it has shown good agreement with manual measurements [38] as well as good reliability in aging cohorts [39]. In addition, we obtained the hippocampal volumes from the same processing stream.

\section{Statistics}

In order to test the overall effects of time on cortical thickness, the surface-based data representing thickness change for each subject was fit into a GLM model for group-statistics. We first calculated the mean rate of change between acquisitions for the whole sample, using a one-sample $t$-test model. We then performed vertex-wise correlations and tested interactions to investigate relationships between thickness, CSF markers, and cognition. First, participants were divided into two subgroups according to their levels of CSF $A \beta_{42}$, namely $A \beta+$ $(<600 \mathrm{pg} / \mathrm{mL})$ and $A \beta-(\geq 600 \mathrm{pg} / \mathrm{mL})$. Because several cutoff values of CSF $A \beta_{42}$ levels are described in the literature, ranging from 500 to $650 \mathrm{pg} / \mathrm{mL}[19$, $40-42]$, we used a rather conservative level of 600 $\mathrm{pg} / \mathrm{mL}$, which is in-between the cut-off used in clinical routine in the Clinical Neurochemistry Laboratory and that for amyloid PET positivity [43], and also to accommodate possible variation or bias in the measurements and to ensure a considerable number of subjects within the group of healthy older adults with amyloid positivity.

General linear models (GLM) were used for vertex-wise analyses of cortical thickness change. All statistical maps were corrected for family wiseerror (FWE) using precomputed simulated data in FreeSurfer [44]. Significance threshold was set at a $p$-value of 0.05 , both for the initial thresholding of the maps and for the identification of significant clusters. Age and gender were used as covariates in all the surface-based analyses. We first calculated the mean rate of change between acquisitions as symmetrized annual percent change, and then tested whether this was different from zero. Additional GLMs were used to compare rate of change between the amyloid groups, and to test the relationship between thickness change and each variable of interest, i.e., the two CSF biomarkers and longitudinal change in each cognitive test score. To test 
interactions, we used a model with two groups (i.e., subjects being positive or negative for $\mathrm{A} \beta_{42}$ ) and CSF YKL-40 levels or the cognitive score as the continuous variable, and tested whether the slopes of the groups differed.

We tested for group differences in 2-year change in the cognitive scores by use of $t$-tests. We also used $t$-test to test for group differences in education and $\mathrm{WMH}$, and used partial correlations, controlling for age and gender, to test whether these variables were related to CSF levels of YKL-40, cognitive scores, or mean cortical thickness. Statistics on non-imaging data were performed with SPSS and MATLAB tools.

\section{RESULTS}

\section{Demographics and cognitive data}

A total of 27 subjects were included in the A $\beta+$ group and 62 in the $A \beta$ - group. Principal component analysis of cognition change depicted three components, from which the first one, used as global cognition change factor (COGfac-change) accounted for $42.63 \%$ of the total variance. There were no group differences in the rates of change in any of the cognitive measures or in baseline CSF YKL40 and P-Tau or T-tau levels. The results of the TMTB at the second time point differed between groups $(t=2.54, p=0.016)$. However, the rates of change within this measure were not significantly

A.

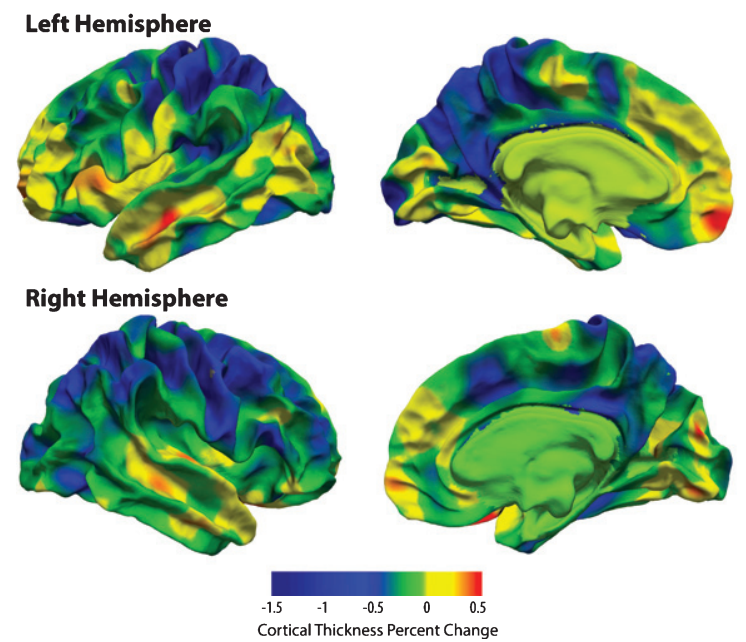

different $(t=1.3, p=0.16)$. Years of education did not differ between groups $(t=1.43, p=0.155)$ and did not correlate with CSF levels, cognitive scores or mean thickness values ( $p>0.05$ in all tests). In addition, there were no differences between groups in $\mathrm{WMH}$ at either time point or in percent change (Table 1), and WMH did not correlate with CSF YKL40 levels or any of the cognitive measures used (all $p$ 's $>0.05$ ).

\section{Mean longitudinal changes in cortical thickness}

We observed an average rate of cortical thinning of $0.53 \%$ (SD: $1.0 \%$ ) per year in the full sample. General thinning was observable across large regions of the cortex (Fig. 1). Reductions in cortical thickness were significant (FWE corrected level of $p<0.05$ ) in several clusters located bilaterally in superior parietal, supramarginal, precuneus, isthmus cingulate posterior cingulate, postcentral and paracentral cortices, in the right hemisphere including the superior frontal, caudalmiddlefrontal, inferior parietal, precentral fusiform, and parahippocampal cortices, as well as in left lingual cortex.

\section{$A \beta_{42}$ and cortical thinning}

Overall change in cortical thickness did not differ between $A \beta+$ and $A \beta-$ participants $(-0.50 \%$ and $-0.54 \%$, respectively, $t=0.11, p=0.91)$. We used a vertex-wise GLM on the surface to investigate

B.
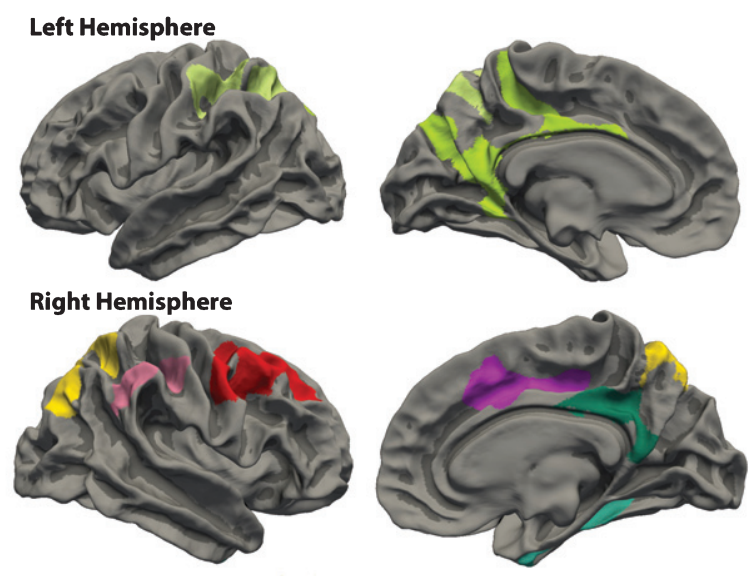

corrected $p<0.05$

Fig. 1. A) Mean effects of time on cortical thickness within the full sample. Percentage of thickness change measured between baseline and the 2-years scan. Green-blue areas indicate decreases over time and yellow-red indicate increases (see legend). B) Significant clusters showing the effect of time on cortical thickness after correction for multiple comparisons $(p<0.05)$. 

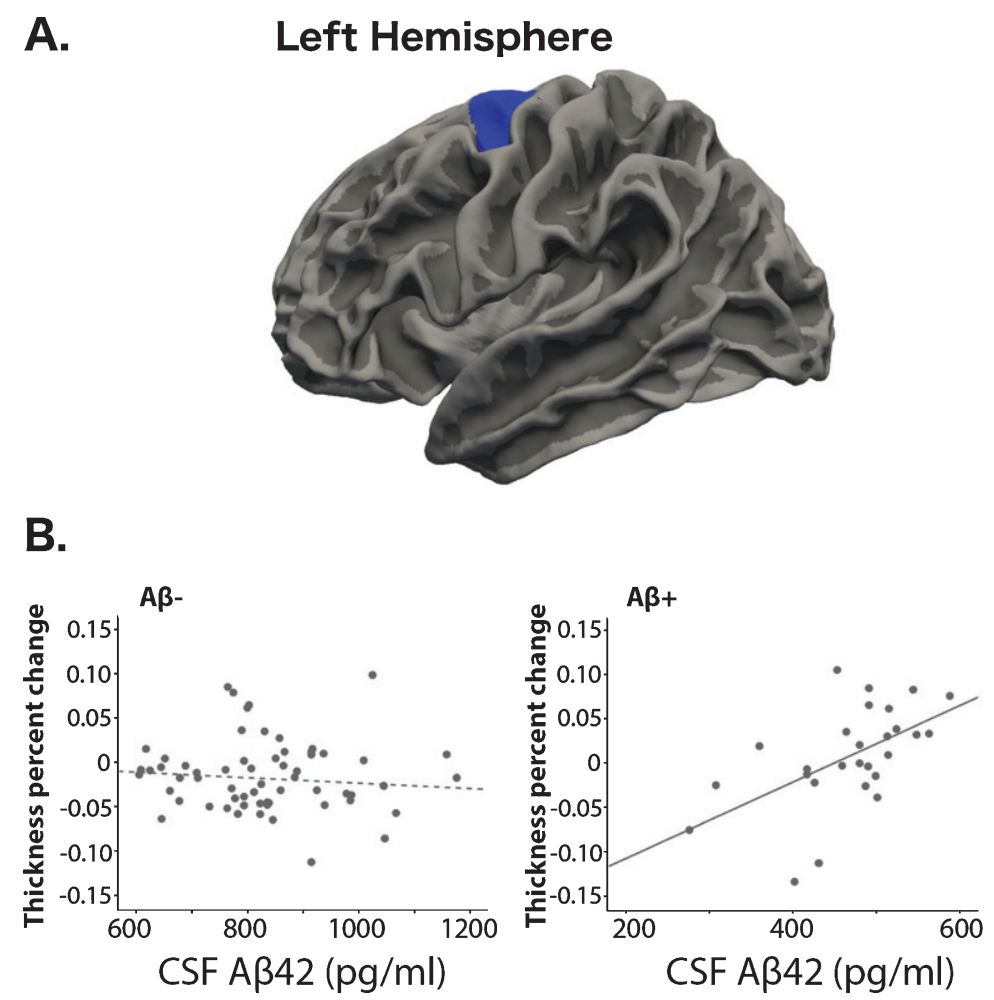

Fig. 2. A) Region with a group-interaction with $A \beta_{42}$ levels and cortical thickness change. B) Scatter plots showing the relationship between thickness change and $A \beta_{42}$ levels for the two groups separately.

the effect of CSF $A \beta_{42}$ levels on regional thickness change. Using a between-group comparison, we found that regional change in thickness did not differ between groups. We then created a design with separate slopes for the relationship between $A \beta$ and thickness change for the $A \beta+$ versus the $A \beta-$ groups. We found a cluster where this interaction was significant, covering part of the left precentral, left caudal middle frontal and superior frontal cortex $(p<0.05$, FWE corrected). In this region, there was a negative relationship between CSF $A \beta_{42}$ levels and the reduction of cortical thickness in the $A \beta+$ group, but not in the $A \beta$ - group (Fig. 2).

\section{$A \beta_{42}$ and cognition}

Using partial correlations and $A \beta_{42}$ as a continuous variable, we did not find any relationship between CSF levels of $A \beta_{42}$ and the global cognitive change or change in any of the specific cognitive measures. More specifically, the correlation with TMTB-change, a test that showed differences between groups at the second time point, was not significant either $(r=-0.173, p=0.115)$.

\section{$Y K L-40, A \beta_{42}$, and brain measures}

We did not find any relationship between YKL40 levels and change in cortical thickness or CSF $A \beta_{42}$ levels. In addition, there were no interactions between these three measures. Even if it was not the aim of the current study, we observed that YKL-40 and hippocampal atrophy correlated on a trend level only $(\mathrm{A} \beta+p=0.06 / \mathrm{A} \beta-p=0.09)$. Therefore, more thorough analyses will be the focus of future studies.

\section{YKL-40 and cognition}

Change in the animal naming test (ANT-change) correlated with CSF YKL-40 levels within the whole sample, in that higher YKL-40 levels were related to more decline in the test scores $(r=-0.28, p=0.012)$ (Fig. 3A). Note that we observed rates of change above zero due to practice effects [45]. No significant correlations between YKL-40 and the other cognitive measures were found, but a trend was observed between YKL-40 and global cognition change (COGfac-change, $r=-0.185, p=0.096$ ).

Finally, we explored correlations with the CSF YKL-40 levels and cognition in $\mathrm{A} \beta+$ and 
A.

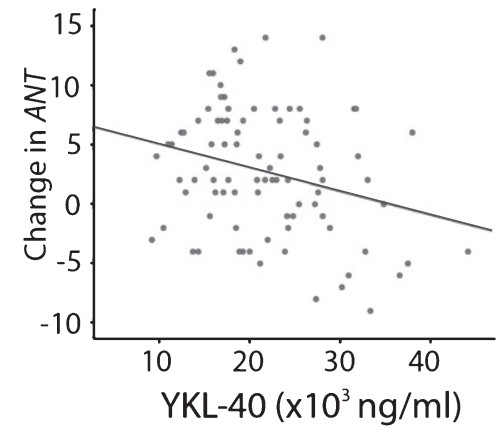

B.

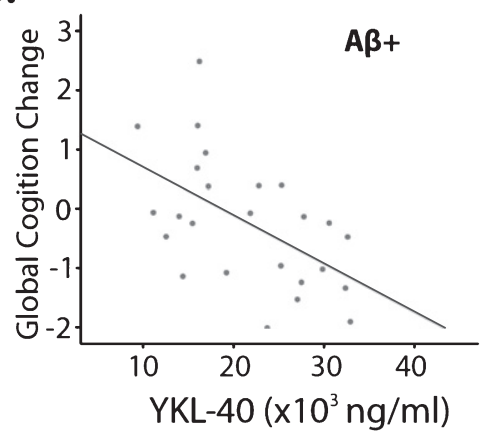

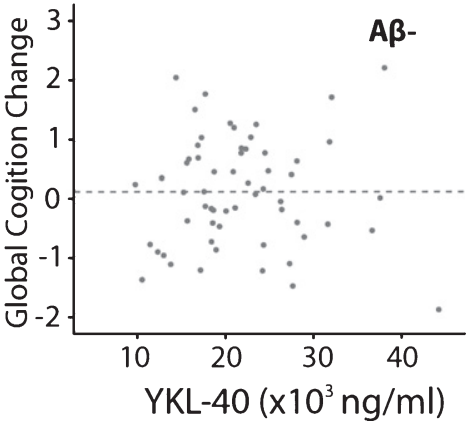

Fig. 3. CSF YKL-40 levels and cognition. A) YKL-40 and changes in animal naming test in the full sample. B) Correlation between YKL-40 and global cognition in $A \beta+$ and $A \beta$ - groups of participants.

$A \beta-$ participants. In the $A \beta+$ group, we found a correlation between levels of YKL-40 and the COGfac-change ( $r=-0.58, p=0.004$, see Fig. 3B). This correlation was not found in the $\mathrm{A} \beta-$ group $(r=-0.008, p=0.955)$. These correlations were significantly different, as evidenced by use of the Fisher $\mathrm{r}$-to- $\mathrm{z}$ transformation $(\mathrm{z}=2.72, p=0.0065$, two-tailed).

\section{Cortical thickness and cognition}

Longitudinal change in cortical thickness correlated positively with change in the results of the animal naming test $(p<0.05)$, meaning that more thinning was associated with more decline in test scores. We found two significant clusters located in the right hemisphere, covering parts of the supramarginal, postcentral, and inferior parietal cortices (Fig. 4).

\section{Exploring possible single-subject effects}

We excluded a single subject having MMSE of 24 at the second timepoint, which was classified into the $\mathrm{A} \beta-$ group and we observed that the main results of the study were preserved. Specifically, the correlations between CSF YKL-40 levels and the ANT scores $(r=-0.281, p=0.011)$, and with the COGfacchange in the $\mathrm{A} \beta$ - group $(r=0.103, p=0.446)$ were preserved. In addition, the vertex-wise comparisons performed with Freesurfer yielded similar results.

\section{DISCUSSION}

In the present study, we showed that neuroinflammation, as indexed by CSF levels of YKL-40, were associated with reduced cognitive function two years

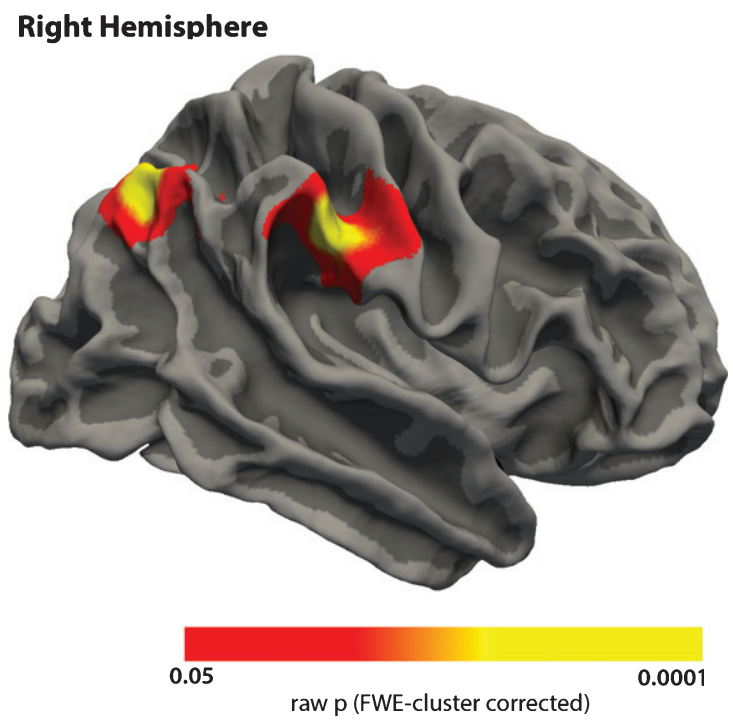

Fig. 4. Correlation between change in cortical thickness and change in the results of the animal naming test. Red-yellow areas indicate that changes in thickness and changes in cognition have the same direction. Only results within significant clusters are shown.

later in cognitively normal older adults who were positive for $A \beta_{42}$. Intriguingly, no relationship was observed for $A \beta$ negative participants. As CSF levels of $A \beta_{42}$ and YKL-40 were not related, this could indicate that these biomarkers are indicators of distinct processes in the brain that have additive detrimental effects on cognitive function in older adults at risk for $\mathrm{AD}$. In addition, we found that CSF $\mathrm{A} \beta_{42}$ levels correlated with cortical atrophy only in amyloid positive participants. Seen together, these results indicate that mechanisms for brain atrophy and cognitive decline might be different in older adults that are positive in comparison with those negative for amyloid, underscoring the need to understand how amyloid relates 
to brain atrophy in cognitively normal older adults. The implications of the results are discussed below.

\section{Age-related cortical thinning}

We observed a pattern of age-related cortical thinning from baseline to the 2-year follow-up acquisition. This pattern is in accordance with what has been reported in other studies of healthy older adults and justifies the suitability of our sample to study the effect of CSF markers in this population. Higher rates of thinning were observed in areas corresponding to the default mode network, a system that is functionally altered in aging [46]. Interestingly, the higher vulnerability to cortical atrophy in areas of the DMN healthy aging has been already discussed elsewhere [47].

\section{Neuroinflammation and cognition in $A \beta+$ older adults}

Here we used CSF YKL-40 levels as a measure of neuroinflammation and we found that increased levels of this biomarker were correlated with global changes in cognition in the $A \beta+$ group, indicating that neuroinflammation might play an important role for cognitive changes in older adults with altered amyloid status. In addition, increasing age has been associated with increased microglial activation [9] increased levels of CSF YKL-40 are observed in AD patients $[10,13,16]$ but this is not invariably found $[17,18]$. However, no studies have previously tested whether this biomarker is increased also in healthy older adults with altered amyloid levels. Intriguingly, our result suggests that even if the level of neuroinflammation as indexed by YKL-40 does not vary as a function of amyloid accumulation, the ability of the brain to cope with the inflammation is different depending on the subject amyloid status. Thus, inflammation is associated with cognitive reduction over time in the presence of higher amyloid levels only, with the lower risk older adults probably being able to better cope with the burden of neuroinflammation. This is not an on-off mechanism, however, as we found that changes in the animal naming test correlated with YKL-40 levels also within the full sample. Thus, the present results indicate that YKL40 indexes neuroinflammatory processes that have mild but nevertheless detrimental effects on cognitive function in older adults, and that these detrimental effects are significantly larger in participants with increased $A \beta_{42}$ levels.

\section{Cortical thinning at the different $C S F A \beta_{42}$ levels}

In addition to the $A \beta$-dependent effects of neuroinflammation on cognition, we found that decreased CSF $A \beta_{42}$ correlated with cortical thinning in the $\mathrm{A} \beta+$ participants. This further indicate that there might be degenerative processes occurring at this narrow range of $A \beta_{42}$ levels even in individuals free from clinical symptoms. This pattern has previously been described within the ADNI cohort of normal older adults $[24,25]$. These two studies found that only under a certain threshold of $A \beta_{42}$ levels in CSF, $A \beta_{42}$ correlated with accelerated brain atrophy. The present study demonstrates this pattern in a different sample than the ADNI database. Taken together, these findings indicate that amyloid positive older adults are less able to cope with processes in the brain that might otherwise not be as harmful in older adults without evidence of brain amyloid accumulation. Previous studies have reported that the anatomical localization of $A \beta$-atrophy relationships tends to vary and seems to be rather widely distributed (for a review, see [47]). This fits well with the fact that amyloid accumulation in the cortex [48] is less anatomically specific than for instance neurofibrillary tangles, which initially is restricted to the medial temporal cortex. In the present study, the stronger relationship between $A \beta_{42}$ and cortical thinning in the $A \beta_{42}+$ group compared with the $A \beta_{42}$ - groups was seen in the motor and premotor cortices, overlapping reported effects from previous studies [21]. However, given the generally widespread distribution of effects in previous studies, we advise against putting too much weight on the precise anatomical location of this effect.

\section{Neuroinflammation, amyloid, and changes in cognition}

Previous literature has described null or relatively weak relationships between common CSF AD biomarkers such as $A \beta_{42}$ and memory/cognition in healthy elderly participants $[26,47,49]$. This has encouraged the community to study the roles of alternative markers in addition to amyloid to better explain cognitive reductions and brain atrophy in aging. Our results, though being moderate in strength, indicate that YKL-40 is one such marker, primarily when seen in coexistence with altered levels of established risk markers such as $A \beta_{42}$. This fits with theories of amyloid and neuroinflammation working together to cause cognitive decline and brain atrophy in older adults with neurodegenerative conditions such as 
$\mathrm{AD}$ [6], even if the evidence for these theories in humans is scarce and to a large extent based on genetic studies. Further, additive roles of amyloid accumulation and neuroinflammation have not been tested in cognitively normal older adults. The results of the present study suggest that effects of inflammation on cognitive decline in older adults without dementia may partly depend on amyloid status.

Adding to this, we found that decline in the same cognitive test also correlated with longitudinal cortical atrophy. Participants with less capability to maintain or improve performance on the animal naming test showed more decreases in cortical thickness. These results are in accordance with previous studies showing correlations between longitudinal changes in brain structure and changes in cognition [50-52].

Although being related with reductions in cognition, CSF YKL-40 levels did not correlate with cortical thinning. Some previous studies have reported correlations between thinner cortex and higher YKL-40 levels $[14,15]$ or other markers of inflammation [5], while others did not find relationships with measures of brain structures such as hippocampal volume [53]. The studies mentioned above were all cross-sectional, in contrast to the present longitudinal design. Further, these former studies included patients with mild cognitive impairment or AD, thus not being fully comparable. Still, when the present results are seen in contrast to the identified relationship between amyloid levels and cortical thinning in the amyloid positive participants, it is evident that the relationship between amyloid levels, neuroinflammation, and brain atrophy is complex. To get a deeper understanding of the mechanisms at play, we will likely also need to include additional biomarkers as well as genetic factors in the analyses.

We performed several tests to evaluate the effect of possible factors confounding our results. We first confirmed that excluding one subject with MMSE of 24 at follow-up did not change the findings, and we found that variables such as WMH or years of education were not correlated with CSF markers or cognition.

However, other neuropathological processes than $A \beta$ could be associated with inflammation even in cognitively well-functioning older adults, which should be focus of future studies. Similarly, even if we did not find any relationship between WMH and the CSF measures or cognitive scores, we believe that more accurate measures of white matter lesions can be used to evaluate in a deeper way the effect of cerebrovascular alterations related to CSF markers in aging. Finally, it has been reported that education, and more generally, cognitive reserve, has an important impact on the aging brain, and that it may interact with cortical thickness, CSF biomarkers, and cognition [54]. However, in our study, years of education were not correlated with cognition or CSF scores.

Our study has several limitations. We report significant correlations at the uncorrected level of $p<0.05$ and therefore our results need to be considered exploratory. However, taking in account the novelty of the subject and the reduced number of related studies, we believe that our findings add relevant information to the field. In addition, as mentioned, further studies should include a wider range of biomarkers, as well as other variables, including genetic and environmental factors and a higher number of data-points. In this sense, we tested whether years of education had an impact on our results and we did not find significant correlation. However, we believe that further studies are needed to investigate these factors in more detail. Similarly, hippocampal atrophy was related to YKL-40 on a trend level, and these results will be followed up in future studies with more in-depth analyses. Another limitation refers to the characteristics of our sample (participants undergoing spinal surgery), which may be not fully representative of a normal aging population. However, the cognitive scores did not indicate cognitive decline, and the rate of change in cortical thickness, as well as the anatomical pattern of cortical thinning, was comparable to those commonly observed in other samples of healthy aging [50]. We believe this supports the validity of the findings.

To conclude, one important theory holds that sustained inflammatory responses to amyloid accumulation increase brain atrophy in $\mathrm{AD}[6,55]$, and the increased levels of neuroinflammation observed also in cognitively normal older adults make it important to investigate amyloid-inflammation relationships also in non-demented. Here we found that YKL-40, as a measure of neuroinflammation, correlated with global cognitive decline especially in amyloid positive older adults. In contrast, CSF $\mathrm{A} \beta_{42}$ levels per se did not correlate with cognitive decline, but still were related to brain atrophy in amyloid positive participants. Put together, these findings highlight the need for considering how newer biomarkers interact with the established ones in the study of cognitive and brain changes in aging. This may provide better understanding of the processes that are crucial 
in the transitional phase between normal aging and neurodegeneration.

\section{ACKNOWLEDGMENTS}

The project is funded by the Norwegian National Association for Public Health, Norway. Further, we have received funding from Innlandet Hospital Trust, the Knut and Alice Wallenberg Foundation, the Swedish Research Council, and the Torsten Söderberg Foundation at the Royal Swedish Academy of Sciences. The authors would also like to thank the study participants.

The funding sources had no role in the study design; in the collection, analysis and interpretation of the data; in the writing of the report; or in the decision to submit the article for publication.

Authors' disclosures available online (http://j-alz. com/manuscript-disclosures/16-1146r2).

\section{REFERENCES}

[1] Blennow K, Hampel H, Weiner M, Zetterberg H (2010) Cerebrospinal fluid and plasma biomarkers in Alzheimer disease. Nat Rev Neurol 6, 131-144.

[2] Mormino EC (2014) The relevance of beta-amyloid on markers of Alzheimer's disease in clinically normal individuals and factors that influence these associations. Neuropsychol Rev 24, 300-312.

[3] Blennow K, Mattsson N, Schöll M, Hansson O, Zetterberg H (2015) Amyloid biomarkers in Alzheimer's disease. Trends Pharmacol Sci 36, 297-309.

[4] Chételat G, Ossenkoppele R, Villemagne VL, Perrotin A, Landeau B, Mézenge F, Jagust WJ, Dore V, Miller BL, Egret S, Seeley WW, van der Flier WM, La Joie R, Ames D, van Berckel BNM, Scheltens P, Barkhof F, Rowe CC, Masters CL, de La Sayette V, Bouwman F, Rabinovici GD (2016) Atrophy, hypometabolism and clinical trajectories in patients with amyloid-negative Alzheimer's disease. Brain 139(Pt 9), 2528-2539.

[5] Fleischman DA, Arfanakis K, Kelly JF, Rajendran N, Buchman AS, Morris MC, Barnes LL, Bennett DA (2010) Regional brain cortical thinning and systemic inflammation in older persons without dementia. J Am Geriatr Soc 58, 1823-1825.

[6] Heneka MT, Carson MJ, El Khoury J, Landreth GE, Brosseron F, Feinstein DL, Jacobs AH, Wyss-Coray T, Vitorica J, Ransohoff RM, Herrup K, Frautschy SA, Finsen B, Brown GC, Verkhratsky A, Yamanaka K, Koistinaho J, Latz E, Halle A, Petzold GC, Town T, Morgan D, Shinohara ML, Perry VH, Holmes C, Bazan NG, Brooks DJ, Hunot S, Joseph B, Deigendesch N, Garaschuk O, Boddeke E, Dinarello CA, Breitner JC, Cole GM, Golenbock DT, Kummer MP (2015) Neuroinflammation in Alzheimer's disease. Lancet Neurol 14, 388-405.

[7] Akiyama H, Barger S, Barnum S, Bradt B, Bauer J, Cole GM, Cooper NR, Eikelenboom P, Emmerling M, Fiebich BL, Finch CE, Frautschy S, Griffin WS, Hampel H, Hull M, Landreth G, Lue L, Mrak R, Mackenzie IR,
McGeer PL, O'Banion MK, Pachter J, Pasinetti G, PlataSalaman C, Rogers J, Rydel R, Shen Y, Streit W, Strohmeyer R, Tooyoma I, Van Muiswinkel FL, Veerhuis R, Walker D, Webster S, Wegrzyniak B, Wenk G, Wyss-Coray T (2000) Inflammation and Alzheimer's disease. Neurobiol Aging 21, 383-421.

[8] Lee JW, Lee YK, Yuk DY, Choi DY, Ban SB, Oh KW, Hong JT (2008) Neuro-inflammation induced by lipopolysaccharide causes cognitive impairment through enhancement of beta-amyloid generation. J Neuroinflammation 5, 37.

[9] Costello DA, Keenan K, McManus RM, Falvey A, Lynch MA (2016) The age-related neuroinflammatory environment promotes macrophage activation, which negatively impacts synaptic function. Neurobiol Aging $\mathbf{4 3}$, 140-148.

[10] Craig-Schapiro R, Perrin RJ, Roe CM, Xiong C, Carter D, Cairns NJ, Mintun MA, Peskind ER, Li G, Galasko DR, Clark CM, Quinn JF, D’Angelo G, Malone JP, Townsend RR, Morris JC, Fagan AM, Holtzman DM (2010) YKL40: A novel prognostic fluid biomarker for preclinical Alzheimer's disease. Biol Psychiatry 68, 903-912.

[11] Rosén C, Andersson C-H, Andreasson U, Molinuevo JL, Bjerke M, Rami L, Lladó A, Blennow K, Zetterberg H (2014) Increased levels of chitotriosidase and YKL-40 in cerebrospinal fluid from patients with Alzheimer's disease. Dement Geriatr Cogn Dis Extra 4, 297-304.

[12] Antonell A, Mansilla A, Rami L, Lladó A, Iranzo A, Olives J, Balasa M, Sánchez-Valle R, Molinuevo JL (2014) Cerebrospinal fluid level of YKL-40 protein in preclinical and prodromal Alzheimer's disease. J Alzheimers Dis 42, 901-908.

[13] Janelidze S, Hertze J, Zetterberg H, Landqvist Waldö M, Santillo A, Blennow K, Hansson O (2016) Cerebrospinal fluid neurogranin and YKL-40 as biomarkers of Alzheimer's disease. Ann Clin Transl Neurol 3, 12-20.

[14] Alcolea D, Vilaplana E, Pegueroles J, Montal V, Sánchez-Juan P, González-Suárez A, Pozueta A, RodríguezRodríguez E, Bartrés-Faz D, Vidal-Piñeiro D, GonzálezOrtiz S, Medrano S, Carmona-Iragui M, Sánchez-Saudinós M, Sala I, Anton-Aguirre S, Sampedro F, MorenasRodríguez E, Clarimón J, Blesa R, Lleó A, Fortea J (2015) Relationship between cortical thickness and cerebrospinal fluid YKL-40 in predementia stages of Alzheimer's disease. Neurobiol Aging 36, 2018-2023.

[15] Gispert JD, Monté GC, Falcon C, Tucholka A, Rojas S, Sánchez-Valle R, Antonell A, Lladó A, Rami L, Molinuevo JL (2016) CSF YKL-40 and pTau181 are related to different cerebral morphometric patterns in early AD. Neurobiol Aging 38, 47-55.

[16] Olsson B, Lautner R, Andreasson U, Öhrfelt A, Portelius E, Bjerke M, Hölttä M, Rosén C, Olsson C, Strobel G, Wu E, Dakin K, Petzold M, Blennow K, Zetterberg H (2016) CSF and blood biomarkers for the diagnosis of Alzheimer's disease: A systematic review and meta-analysis. Lancet Neurol 15, 673-684.

[17] Hellwig K, Kvartsberg H, Portelius E, Andreasson U, Oberstein TJ, Lewczuk P, Blennow K, Kornhuber J, Maler JM, Zetterberg H, Spitzer P (2015) Neurogranin and YKL-40: Independent markers of synaptic degeneration and neuroinflammation in Alzheimer's disease. Alzheimers Res Ther 7, 74.

[18] Mattsson N, Tabatabaei S, Johansson P, Hansson O, Andreasson U, Månsson J-E, Johansson J-O, Olsson B, Wallin A, Svensson J, Blennow K, Zetterberg H (2011) Cerebrospinal fluid microglial markers in Alzheimer's 
disease: Elevated chitotriosidase activity but lack of diagnostic utility. Neuromolecular Med 13, 151-159.

[19] Fagan AM, Head D, Shah AR, Marcus D, Mintun M, Morris JC, Holtzman DM (2009) Decreased cerebrospinal fluid Abeta(42) correlates with brain atrophy in cognitively normal elderly. Ann Neurol 65, 176-183.

[20] Schott JM, Bartlett JW, Fox NC, Barnes J, Alzheimer's Disease Neuroimaging Initiative Investigators (2010) Increased brain atrophy rates in cognitively normal older adults with low cerebrospinal fluid Aß1-42. Ann Neurol 68, 825-834.

[21] Tosun D, Schuff N, Truran-Sacrey D, Shaw LM, Trojanowski JQ, Aisen P, Peterson R, Weiner MW (2010) Relations between brain tissue loss, CSF biomarkers, and the ApoE genetic profile: A longitudinal MRI study. Neurobiol Aging 31, 1340-1354.

[22] Chételat G, Villemagne VL, Pike KE, Baron J-C, Bourgeat P, Jones G, Faux NG, Ellis KA, Salvado O, Szoeke C, Martins RN, Ames D, Masters CL, Rowe CC (2010) Larger temporal volume in elderly with high versus low betaamyloid deposition. Brain 133, 3349-3358.

[23] Fortea J, Sala-Llonch R, Bartrés-Faz D, Lladó A, SoléPadullés C, Bosch B, Antonell A, Olives J, Sanchez-Valle R, Molinuevo JL, Rami L (2011) Cognitively preserved subjects with transitional cerebrospinal fluid $\beta$-amyloid 1-42 values have thicker cortex in Alzheimer's disease vulnerable areas. Biol Psychiatry 70, 183-190.

[24] Becker JA, Hedden T, Carmasin J, Maye J, Rentz DM, Putcha D, Fischl B, Greve DN, Marshall GA, Salloway S, Marks D, Buckner RL, Sperling RA, Johnson KA (2011) Amyloid- $\beta$ associated cortical thinning in clinically normal elderly. Ann Neurol 69, 1032-1042.

[25] Fjell AM, Walhovd KB, Fennema-Notestine C, McEvoy LK, Hagler DJ, Holland D, Blennow K, Brewer JB, Dale AM, Alzheimer's Disease Neuroimaging Initiative (2010) Brain atrophy in healthy aging is related to CSF levels of Aß1-42. Cereb Cortex 20, 2069-2079.

[26] Hedden T, Oh H, Younger AP, Patel TA (2013) Metaanalysis of amyloid-cognition relations in cognitively normal older adults. Neurology 80, 1341-1348.

[27] Morris JC, Heyman A, Mohs RC, Hughes JP, van Belle G, Fillenbaum G, Mellits ED, Clark C (1989) The Consortium to Establish a Registry for Alzheimer's Disease (CERAD). Part I. Clinical and neuropsychological assessment of Alzheimer's disease. Neurology 39, 1159-1165.

[28] Spreen O, Strauss E (1991) A compendium of neuropsychological tests: Administration, norms, and commentary, Oxford University Press, New York.

[29] Reitan RM (1955) The relation of the trail making test to organic brain damage. J Consult Psychol 19, 393-394.

[30] Folstein MF, Folstein SE, McHugh PR (1975) "Mini-mental state". A practical method for grading the cognitive state of patients for the clinician. J Psychiatr Res 12, 189-198.

[31] Shulman KI (2000) Clock-drawing: Is it the ideal cognitive screening test? Int J Geriatr Psychiatry 15, 548-561.

[32] Kendrick DC, Gibson AJ, Moyes IC (1979) The revised kendrick battery: Clinical studies. Br J Soc Clin Psychol 18, 329-340.

[33] Dale AM, Fischl B, Sereno MI (1999) Cortical surfacebased analysis. I. Segmentation and surface reconstruction. Neuroimage 9, 179-194.

[34] Fischl B, Salat DH, Busa E, Albert M, Dieterich M, Haselgrove C, van der Kouwe A, Killiany R, Kennedy D, Klaveness S, Montillo A, Makris N, Rosen B, Dale AM (2002) Whole brain segmentation: Automated labeling of neuroanatomical structures in the human brain. Neuron $\mathbf{3 3}$, 341-355.

[35] Jovicich J, Marizzoni M, Sala-Llonch R, Bosch B, BartrésFaz D, Arnold J, Benninghoff J, Wiltfang J, Roccatagliata L, Nobili F, Hensch T, Tränkner A, Schönknecht P, Leroy M, Lopes R, Bordet R, Chanoine V, Ranjeva J-P, Didic M, Gros-Dagnac H, Payoux P, Zoccatelli G, Alessandrini F, Beltramello A, Bargalló N, Blin O, Frisoni GB (2013) Brain morphometry reproducibility in multi-center 3T MRI studies: A comparison of cross-sectional and longitudinal segmentations. Neuroimage $\mathbf{8 3}, 472-484$.

[36] Reuter M, Schmansky NJ, Rosas HD, Fischl B (2012) Within-subject template estimation for unbiased longitudinal image analysis. Neuroimage 61, 1402-1418.

[37] Reuter M, Fischl B (2011) Avoiding asymmetry-induced bias in longitudinal image processing. Neuroimage 57, 1921.

[38] Smith EE, Salat DH, Jeng J, McCreary CR, Fischl B, Schmahmann JD, Dickerson BC, Viswanathan A, Albert MS, Blacker D, Greenberg SM (2011) Correlations between MRI white matter lesion location and executive function and episodic memory. Neurology 76, 1492-1499.

[39] Olsson E, Klasson N, Berge J, Eckerström C, Edman A, Malmgren H, Wallin A, Malmgren H, Wallin A (2013) White matter lesion assessment in patients with cognitive impairment and healthy controls: Reliability comparisons between visual rating, a manual, and an automatic volumetrical MRI method-the Gothenburg MCI study. J Aging Res 2013, 198471.

[40] Mulder C, Verwey NA, van der Flier WM, Bouwman FH, Kok A, van Elk EJ, Scheltens P, Blankenstein MA (2010) Amyloid-beta(1-42), total tau, and phosphorylated tau as cerebrospinal fluid biomarkers for the diagnosis of Alzheimer disease. Clin Chem 56, 248-253.

[41] Niemantsverdriet E, Goossens J, Struyfs H, Martin J-J, Goeman J, De Deyn PP, Vanderstichele H, Engelborghs S (2016) Diagnostic impact of cerebrospinal fluid biomarker (pre)analytical variability in Alzheimer's disease. J Alzheimers Dis 51, 97-106.

[42] Zwan MD, Rinne JO, Hasselbalch SG, Nordberg A, Lleó A, Herukka S-K, Soininen H, Law I, Bahl JMC, Carter SF, Fortea J, Blesa R, Teunissen CE, Bouwman FH, van Berckel BNM, Visser PJ (2016) Use of amyloid-PET to determine cutpoints for CSF markers: A multicenter study. Neurology 86, $50-58$.

[43] Palmqvist S, Zetterberg H, Blennow K, Vestberg S, Andreasson U, Brooks DJ, Owenius R, Hägerström D, Wollmer P, Minthon L, Hansson O (2014) Accuracy of brain amyloid detection in clinical practice using cerebrospinal fluid $\beta$-amyloid 42: A cross-validation study against amyloid positron emission tomography. JAMA Neurol 71, 1282-1289.

[44] Hagler DJ, Saygin AP, Sereno MI (2006) Smoothing and cluster thresholding for cortical surface-based group analysis of fMRI data. Neuroimage 33, 1093-1103.

[45] Galvin JE, Powlishta KK, Wilkins K, McKeel DW, Xiong C, Grant E, Storandt M, Morris JC (2005) Predictors of preclinical Alzheimer disease and dementia: A clinicopathologic study. Arch Neurol 62, 758-765.

[46] Damoiseaux JS, Beckmann CF, Arigita EJ, Barkhof F, Scheltens P, Stam CJ, Smith SM, Rombouts SA (2008) Reduced resting-state brain activity in the "default network" in normal aging. Cereb Cortex 18, 1856-1864.

[47] Fjell AM, McEvoy L, Holland D, Dale AM, Walhovd KB (2014) What is normal in normal aging? Effects of aging, 
amyloid and Alzheimer's disease on the cerebral cortex and the hippocampus. Prog Neurobiol 117, 20-40.

[48] Braak H, Braak E (1997) Frequency of stages of Alzheimerrelated lesions in different age categories. Neurobiol Aging 18, 351-357.

[49] Aschenbrenner AJ, Balota DA, Fagan AM, Duchek JM, Benzinger TLS, Morris JC (2015) Alzheimer disease cerebrospinal fluid biomarkers moderate baseline differences and predict longitudinal change in attentional control and episodic memory composites in the Adult Children Study. J Int Neuropsychol Soc 21, 573-583.

[50] Fjell AM, McEvoy L, Holland D, Dale AM, Walhovd KB (2013) Brain changes in older adults at very low risk for Alzheimer's disease. J Neurosci 33, 8237-8242.

[51] Persson N, Ghisletta P, Dahle CL, Bender AR, Yang Y, Yuan P, Daugherty AM, Raz N (2016) Regional brain shrinkage and change in cognitive performance over two years: The bidirectional influences of the brain and cognitive reserve factors. Neuroimage 126, 15-26.

[52] Rodrigue KM, Raz N (2004) Shrinkage of the entorhinal cortex over five years predicts memory performance in healthy adults. $J$ Neurosci 24, 956-963.
[53] Melah KE, Lu SY-F, Hoscheidt SM, Alexander AL, Adluru N, Destiche DJ, Carlsson CM, Zetterberg H, Blennow K, Okonkwo OC, Gleason CE, Dowling NM, Bratzke LC, Rowley HA, Sager MA, Asthana S, Johnson SC, Bendlin BB (2015) Cerebrospinal fluid markers of Alzheimer's disease pathology and microglial activation are associated with altered white matter microstructure in asymptomatic adults at risk for Alzheimer's disease. J Alzheimers Dis 50, 873886.

[54] Arenaza-Urquijo EM, Molinuevo J-L, Sala-Llonch R, SoléPadullés C, Balasa M, Bosch B, Olives J, Antonell A, Lladó A, Sánchez-Valle R, Rami L, Bartrés-Faz D (2013) Cognitive reserve proxies relate to gray matter loss in cognitively healthy elderly with abnormal cerebrospinal fluid amyloid$\beta$ levels. J Alzheimers Dis 35, 715-726.

[55] Gouwens LK, Makoni NJ, Rogers VA, Nichols MR (2016) Amyloid- $\beta 42$ protofibrils are internalized by microglia more extensively than monomers. Brain Res 1648, 485-495. 